\title{
Seeing in the dark
}

An imaging platform based on stimulated emission helps researchers to lead nonfluorescent chromophores out of the shadows.

For years now, fluorescent reagents and proteins have reigned as the undisputed champions of the biological microscopy world. Lightabsorbing molecules receive a jolt of energy upon illumination at an appropriate wavelength, which kicks an electron in their structure from the relaxed 'ground state' into a transient 'excited state'; fluorescent compounds get their glow when this energy is discharged in the form of an externally detectable photon as the excited electron returns to ground state, a process known as 'spontaneous emission'.

The problem, points out Harvard University's Sunney Xie, is that only a relative handful of molecules truly shine as fluorescence reagents. "Many molecules absorb but do not fluoresce or have undetectable fluorescence, such as hemoglobin, cytochrome, retinal and melanin," he says. "Upon photoexcitation, those molecules quickly return to the ground state not through spontaneous emission but via the release of energy as heat."

As a means to visualize these nonfluorescent chromophores, his team has made use of stimulated emission, a principle described by Albert Einstein nearly a century ago. Xie's system, which he developed along with postdoctoral fellow Wei Min and graduate student Sijia Lu, makes use of two ultrafast lasers. The first fires a pulse that bumps an electron within the target chromophore into an excited state; this is closely followed by a pulse from the second laser, which knocks the electron back into the ground state in a manner that creates an additional photon with the same energy and direction as the initial, stimulatory photons.

This process of light amplification via stimulated emission lies at the core of laser technology and creates a signal boost for otherwise-invisible chromophores. "It's the first time that stimulated emission has been used as a contrast mechanism for microscopy," says Xie, "and this allows us to visualize molecules that were otherwise undetectable before." As an initial proof of concept, they used stimulated emission microscopy to detect the distribution of green fluorescent protein-derived chromophores gtCP and cjBlue in live Escherichia coli and to visualize the indigo byproduct of X-gal cleavage by the enzyme $\beta$-galactosidase-a widely used reporter for gene activity —at otherwise undetectable basal levels of enzyme activity.

Stimulated emission may likewise prove helpful for imaging of fluorescent targets that have a tendency to disappear when they 'blink'. "Green fluorescent proteins will go to a dark state, where they don't emit but still absorb; quantum dots do the same thing," says Xie. "We could now have this new mechanism that allows people to see the dark state."

This method also holds promise for clinical applications. For example, stimulated emission enabled visualization of the uptake of the photosensitizing therapeutic agent toluidine blue $\mathrm{O}$ by cancer cells. Xie's group exploited their capacity to image hemoglobin as a means for visualizing the microvasculature within the ear of a mouse, with individual red blood cells clearly visible.

These preliminary findings establish a wide range of potential applications for stimulated emission microscopy, and Xie is eager to move forward with this platform. "We have collaborators in the skin care industry, and we work with surgeons and neurologists," he says. "The study of melanin in the skin and the distribution of microcapillaries around tumors or neurons [is] based on the contrast of hemoglobin; we'll be able to visualize them with this label-free imaging method and follow them in real time."

\section{Michael Eisenstein}

\section{RESEARCH PAPERS}

Min, W. et al. Imaging chromophores with undetectable fluorescence by stimulated emission microscopy. Nature 461, 1105-1109 (2009). 